\title{
Inflammatory syndromes associated with SARS-CoV-2 infection: dysregulation of the immune response across the age spectrum
}

\author{
Jill E. Weatherhead, ${ }^{1,2,3}$ Eva Clark, ${ }^{1,3,4}$ Tiphanie P. Vogel, ${ }^{5,6}$ Robert L. Atmar, ${ }^{1}$ and Prathit A. Kulkarni ${ }^{1,7}$ \\ 'Department of Medicine, Section of Infectious Diseases, ${ }^{2}$ Department of Pediatrics, Section of Pediatric Tropical Medicine, and ${ }^{3}$ National School of Tropical Medicine, Baylor College of Medicine, Houston, \\ Texas, USA. ${ }^{4}$ Department of Medicine, Section of Health Services Research, Center for Innovations in Quality, Safety, and Effectiveness (IQuESt), Michael E. DeBakey VA Medical Center and Baylor College of \\ Medicine, Houston, Texas, USA. 5Department of Pediatrics, Section of Rheumatology, and 'DDepartment of Medicine, Section of Immunology, Allergy and Rheumatology, Baylor College of Medicine, Houston, \\ Texas, USA. ${ }^{7}$ Medical Care Line, Michael E. DeBakey Veterans Affairs Medical Center, Houston, Texas, USA.
}

c oronavirus disease 2019 (COVID-19) has devastated the global community since the emergence of severe acute respiratory syndrome coronavirus-2 (SARS-CoV-2) in late 2019. Most COVID-19 cases occur in adults, and a subset develop COVID-19-associated hyperinflammatory syndrome (1), resulting in progressive respiratory illness and multiorgan dysfunction. Progression to severe COVID-19 after SARS-CoV-2 exposure is less common in young adults and children. However, since March 2020, areas with high community SARS-CoV-2 transmission have reported increasing cases of a pediatric inflammatory syndrome that occurs weeks to months after initial asymptomatic or mild SARS-CoV-2 infection (2). The US CDC termed this condition multisystem inflammatory syndrome in children (MIS-C, also known as pediatric inflammatory multisystem syndrome temporally associated with SARS-CoV-2 [PIMS-TS] in Europe, where it was first recognized). Since June 2020, a similar syndrome (MIS-A) has been recognized in adults (3). The pathology of MIS$\mathrm{C} / \mathrm{A}$ and its relationship to SARS-CoV-2 infection are not yet known.

Available evidence suggests significant heterogeneity in the human immune response to SARS-CoV-2. In this Viewpoint, we examine the spectrum of SARS$\mathrm{CoV}-2$-associated clinical inflammatory syndromes identified in adult and pediatric populations to date. A better understanding of the immunopathologies and related clinical manifestations of these syndromes is necessary to improving their diagnosis and management.

\section{Severe COVID-19-associated hyperinflammatory syndrome in adults}

While most adults have asymptomatic or mild infections after exposure to SARSCoV-2, those who progress to severe COVID-19 typically have preexisting, proinflammatory conditions, particularly cardiovascular disease, diabetes, and obesity. Severe COVID-19 can be complicated by respiratory failure, cardiac pathology, and thrombotic events. Patients with such complications often have high levels of systemic inflammatory markers (including C-reactive protein [CRP], ferritin, lactate dehydrogenase, and D-dimer), an increased neutrophil-to-lymphocyte ratio, and elevated levels of circulating proinflammatory cytokines and chemokines (4).

These observations suggest that in vulnerable individuals, SARS-CoV-2 triggers a dysregulated inflammatory response, known as COVID-19-associated hyperinflammatory syndrome (1). Indeed, the complications of severe COVID-19 are often due to the sequelae of this hyperinflammation more than direct viral toxicity. Specifically, organ-system damage results from an uncontrolled cytokine cascade similar to that triggered in macrophage activation syndrome (MAS) and secondary hemophagocytic lymphohistiocytosis (HLH). In contrast to MAS and HLH, COVID-19-associated hyperinflammatory syndrome less commonly demonstrates hepatosplenomegaly, alterations in fibrinogen, or elevated serum levels of CD25, making it a distinct inflammatory syndrome

Authorship note: JEW and EC contributed equally to this work.

Conflict of interest: The authors have declared that no conflict of interest exists.

Copyright: (c) 2020, American Society for Clinical Investigation.

Reference information: J Clin Invest. 2020;130(12):6194-6197. https://doi.org/10.1172/JCI145301.

(1). Importantly, lymphocytopenia is an early indicator of impending severe disease. Although the exact cause of lymphocytopenia remains poorly understood, it may be related to immune exhaustion or lymphocyte apoptosis combined with an inability to restore adequate lymphocyte supply (5). This paucity of lymphocytes could be a manifestation of imbalance in the innate and acquired immune responses, resulting in delayed viral clearance, dysregulated interferon production, and hyperstimulated macrophages and neutrophils (Figure 1) (6). Patients with severe COVID-19 demonstrate distinct immunophenotypes, ranging from complete lack of $\mathrm{T}$ or $\mathrm{B}$ cell responses to an overexaggerated activation of $\mathrm{CD}^{+}$ $\mathrm{T}$ cells with hyperactivated and exhausted $\mathrm{CD}^{+} \mathrm{T}$ cells (7). Interestingly, recent data indicate that genetic and acquired defects in type I interferon signaling may predispose individuals to severe COVID-19 (8).

These immunologic observations have informed treatment and research protocols for patients with COVID-19-associated hyperinflammatory syndrome. Immunomodulatory therapies and antiinflammatory agents, including corticosteroids, have become accepted treatments for severe COVID-19. Continued analysis of immune dysregulation during the entire time course of COVID-19 in patients with a range of clinical presentations is critical to optimizing the timing, dosing, safety, and efficacy of these therapies and to developing new therapeutic agents.

\section{A SARS-CoV-2-associated hyperinflammatory syndrome in children}

Children are thought to be less susceptible than adults to SARS-CoV-2 (9), and COVID-19 has been documented much 


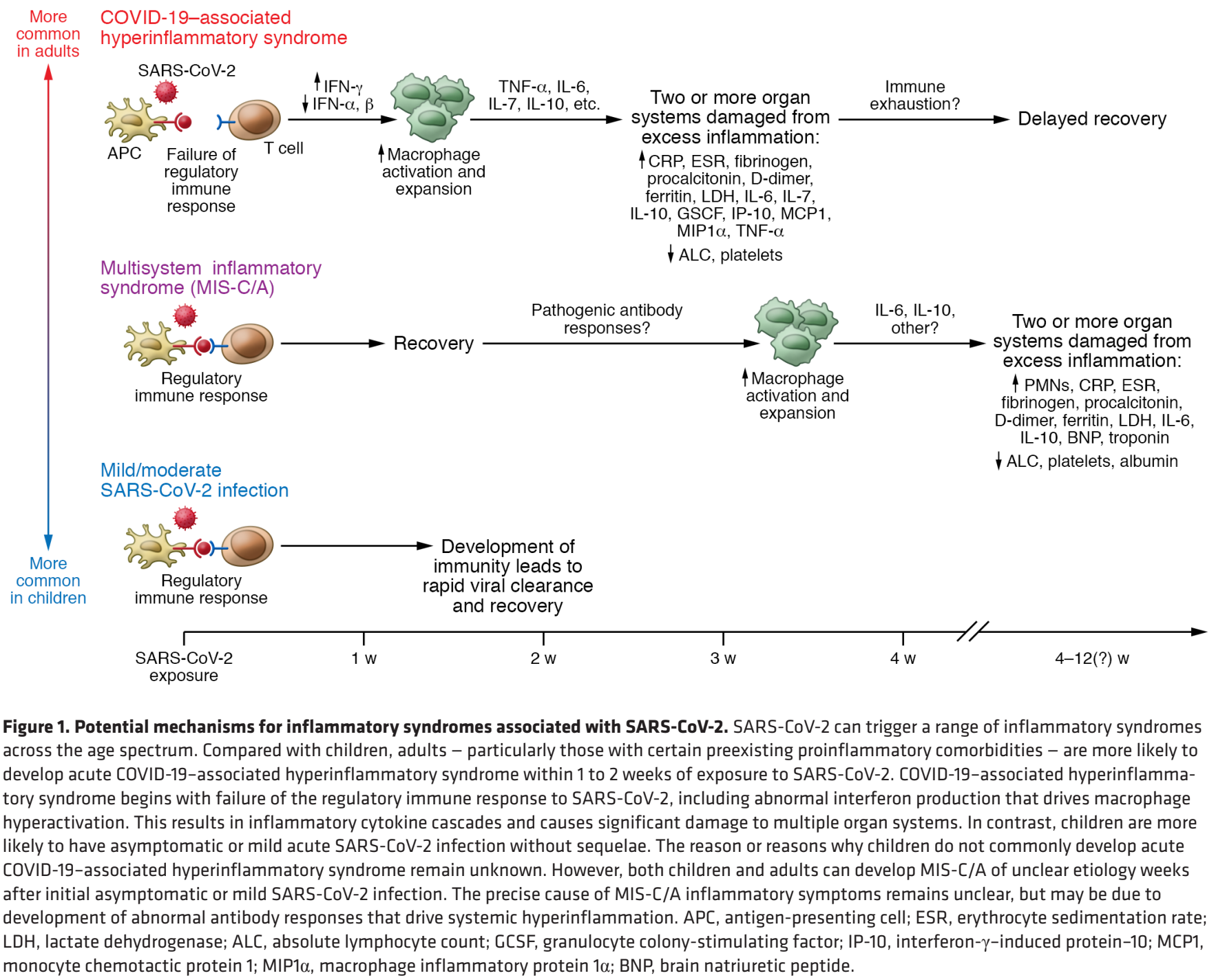

less often in children (10). The vast majority of pediatric SARS-CoV-2 infections are asymptomatic or cause only mild symptoms. In the United States, pediatric hospitalization rates are low (8.0/100,000 persons) compared with those for adults (164.5/100,000 persons) (11). Although one-third of hospitalized children require intensive care (similar to adults), severe COVID-19 and death rarely occur in children and are usually limited to those with underlying health conditions (including obesity) or infants (12).

While the specific mechanism or mechanisms that protect children from severe COVID-19 are unclear (13), there are five leading theories. Compared with older adults, younger individuals may have (a) a more robust innate immune response during early infection that allows for early control of viral replication; (b) a less robust adaptive immune response with reduced production of inflammatory $\mathrm{T}$ cells; (c) preestablished partial protective immunity acquired from relatively recent exposure to other coronaviruses during childhood; (d) differences in expression of the cellular receptor and activator of SARS-CoV-2, which lead to lower susceptibility to pulmonary infection compared with adults; and (e) absence of chronic comorbidities that compromise the integrity of the vascular endothelium (13).

Although the young are less susceptible than older adults to acute, severe COVID-19, cases of a delayed hyperinflammatory syndrome of children known as MIS-C - occurring weeks to months after initial asymptomatic or mild SARS-CoV-2 exposure have been observed in populations experiencing significant SARS-CoV-2 transmission (14).
MIS-C was first identified because of its similarity to other pediatric inflammatory syndromes, such as Kawasaki disease (KD), toxic shock syndrome, and MAS. Like the severe COVID-19 hyperinflammatory syndrome seen in adults, MIS-C is defined by significant systemic inflammation and includes elevated serum levels of CRP, procalcitonin, D-dimer, ferritin, IL-10, and IL-6 along with neutrophilia, lymphocytopenia, and thrombocytopenia (14). Many MIS-C patients have a negative SARS-CoV-2 reverse-transcriptase PCR (RT-PCR) test, but positive SARS-CoV-2 serology (15), specifically positive IgG and negative IgM (16), suggesting that MIS-C develops weeks after initial asymptomatic or mild SARS-CoV-2 infection (Figure 1). The predominance of previously asymptomatic or mild disease in the setting of detectable SARS-CoV-2 antibody at the 
time of MIS-C diagnosis provides early evidence that development of MIS-C results from delayed hyperimmune activation (including potentially pathogenic antibody responses) rather than direct viral damage, although this concept requires further study (17).

Most MIS-C patients present with fever and a spectrum of symptomatology involving multiple organ systems, including cardiovascular, gastrointestinal, and hematologic manifestations. Notably, pulmonary pathology is not a predominant feature. Many MIS-C patients demonstrate a KD-like phenotype, although such presentations often differ from true $\mathrm{KD}$ in several key areas, including older age, prominent gastrointestinal symptoms, higher levels of inflammation, and the presence of thrombocytopenia rather than thrombocytosis $(18,19)$. MIS-C patients can have significant cardiac involvement, manifested as elevations in brain natriuretic peptide and troponin levels, myocarditis, impaired left ventricular function, and coronary artery abnormalities (14). Patients with MIS-C can become critically ill, requiring inotropic and vasopressor support, mechanical ventilation, and extracorporeal membrane oxygenation. Regardless of severity of disease presentation, children with MIS-C appear to respond promptly to relatively short courses of immunomodulatory agents, such as corticosteroids and intravenous immunoglobulin therapy, the latter selected for its well-documented role in $\mathrm{KD}$ treatment. The mortality rate from MIS-C is low (approximately 2\%), but is higher than the reported mortality rates of primary SARS-CoV-2 infection in children (0\%-0.6\%) (15).

\section{An underrecognized, delayed SARS-CoV-2-associated MIS-A}

Since June 2020, a similar, delayed multisystem inflammatory syndrome has been recognized in adults - distinct from acute COVID-19-associated hyperinflammatory syndrome $(3,20)$. This syndrome, MIS-A, is currently defined as an illness requiring hospitalization in a person older than 21 years with a positive test for current or previous SARS-CoV-2 infection within the preceding 12 weeks, severe extrapulmonary organ dysfunction, and laboratory markers consistent with hyperinflammation in the absence of severe respiratory symptoms (3). MIS-A is likely underrecognized; fewer than 30 cases have been reported to date. While clinical features vary, MIS-A most often manifests with cardiac (including arrhythmias, left ventricular heart failure, and elevated troponins), gastrointestinal, dermatologic, and/or neurologic symptoms. Several of the reported MIS-A cases demonstrate features similar to those of $\mathrm{KD}$, a disease that is extremely uncommon among adults. Because they may have a negative SARS-CoV-2 PCR test, it is imperative to evaluate patients suspected to have MIS-A via SARS-CoV-2 serologic assays to establish the diagnosis; the lack of widespread, early serologic testing in many locations likely contributed to delayed recognition of this clinical entity. Patients with MIS-A have been given various therapies, including intravenous immunoglobulin, corticosteroids, and the IL- 6 receptor inhibitor tocilizumab. While most required intensive care, 24 of 27 reported cases survived (3). Similarly to what occurs with MIS-C, the specific pathologic mechanisms driving MIS-A remain unknown, and more data regarding risk factors, clinical characteristics, and outcomes are needed to guide therapeutic interventions.

\section{Tying it all together:} implications of SARS-CoV-2associated hyperinflammation across the age spectrum

The ability of SARS-CoV-2 to trigger inflammatory syndromes in both adults and children is now well established. However, the detailed features that distinguish acute and delayed forms of SARS-CoV-2related inflammation remain unclear. Current adult models of severe COVID-19 suggest early hyperactivation of the immune response, impaired interferon function, and excessive macrophage activation, further complicated by dysregulation of tissue-repair mechanisms and fibrosis. Such acute progression to severe COVID-19 occurs within 1 to 2 weeks of initial SARSCoV-2 exposure and leads to significant morbidity and mortality. In contrast, a delayed inflammatory response, manifesting as the relatively recently defined MIS$\mathrm{C} / \mathrm{A}$, is increasingly recognized weeks to months after asymptomatic or mild SARSCoV-2 infection. Compared with adults with COVID-19-associated hyperinflam- matory syndrome, children and adults with MIS-C/A present more often with gastrointestinal symptoms and less often with respiratory symptoms and thrombotic sequelae. Additionally, those with MIS-C/A are more likely to be previously healthy and less likely to have positive SARS-CoV-2 molecular tests; rather, they typically have serologic evidence of prior exposure. MIS-C/A patients are also more likely to have favorable clinical outcomes compared with adults with COVID-19associated hyperinflammatory syndrome. Despite these differences, the hyperinflammatory states that characterize severe COVID-19 and MIS-C/A do have notable clinical similarities, such as multiorgan dysfunction, profound elevation in inflammatory markers, and significant cardiovascular involvement with evidence of myocardial and coronary artery injury.

Many important questions remain concerning the immunopathogenesis and optimal management of the inflammatory sequelae of SARS-CoV-2 infection across the age spectrum. The clinical and immunologic risk factors that increase susceptibility to acute hyperinflammation and/ or delayed inflammatory sequelae after SARS-CoV-2 exposure are still unclear. Additionally, while direct SARS-CoV-2triggered maladaptive immune cascades likely contribute to the inflammatory damage seen in severe COVID-19, the cause or causes of MIS-C/A are less apparent. One consideration is that SARS-CoV-2induced autoimmunity may play a role in the pathogenesis of MIS-C/A and may explain reports of weeks to months of persistent symptoms after severe COVID-19 (17). Large, prospective, longitudinal studies assessing the development of delayed inflammation and autoimmunity in both children and adults are warranted for addressing these questions. Mapping out the natural progression of the host inflammatory response to SARS-CoV-2 in relation to viral replication and identifying the optimal timing of immune-modulating therapies will lead to improved outcomes in children and adults with COVID-19 and MIS-C/A. Ideally, biomarkers that distinguish between acute and delayed SARS-CoV-2-associated hyperinflammation will be identified to help with treatment decisions and prognostication. Insights derived from such studies will further 
inform adult and pediatric clinical management and research protocols, with each domain being able to utilize breakthroughs from the other.

Address correspondence to: Jill E. Weatherhead, 1120 Bates Ave., Suite 550, Texas Children's Hospital, Houston, Texas 77030, USA. Phone: 832.824.1317; Email: weatherh@bcm.edu.

1. Webb BJ, et al. Clinical criteria for COVID-19associated hyperinflammatory syndrome: a cohort study. Lancet Rheumatol 2020. Published online September 29, 2020. https:// doi.org/10.1016/S2665-99134(20)30343-x. Accessed October 28, 2020.

2. Multisystem Inflammatory Syndrome in Children (MIS-C) Associated with Coronavirus Diease 2019 (COVID-19). https://emergency.cdc.gov/ han/2020/han00432.asp. Accessed Oct 17, 2020.

3. Morris SB, et al. Case series of multisystem inflammatory syndrome in adults associated with SARS-CoV-2 infection - United Kingdom and United States, March-August 2020. MMWR Morb Mortal Wkly Rep. 2020;69(40):1450-1456.

4. Henry BM, de Oliveira MHS, Benoit S, Plebani M, Lippi G. Hematologic, biochemical and immune biomarker abnormalities associated with severe illness and mortality in coronavirus disease 2019 (COVID-19): a meta-analysis. Clin Chem Lab Med. 2020;58(7):1021-1028.

5. Cully M. Immune status could determine efficacy of COVID-19 therapies. Nat Rev Drug Discov. 2020;19(7):431-434.

6. Hadjadj J, et al. Impaired type I interferon activity and inflammatory responses in severe COVID-19 patients. Science. 2020;369(6504):718-724.

7. Mathew D, et al. Deep immune profiling of COVID-19 patients reveals distinct immunotypes with therapeutic implications. Science. 2020;369(6508):eabc8511.

8. Bastard P, et al. Auto-antibodies against type I IFNs in patients with life-threatening COVID-19. Science. 2020;370(6515):eabd4585.

9. Viner RM, et al. Susceptibility to SARS-CoV-2 infection among children adolescents compared with adults: a systematic review meta-analysis. JAMA Pediatr. Published online September 25, 2020. https://doi.org/10.1001/jamapediatrics.2020.4573.

10. Mehta NS, et al. SARS-CoV-2 (COVID-19): What do we know about children? A systematic review. Clin Infect Dis. 2020; ciaa556.

11. Kim L, et al. Hospitalization rates and characteristics of children aged $<18$ years hospitalized with laboratory-confirmed COVID-19 - COVIDNET, 14 states, March 1-July 25, 2020. MMWR Morb Mortal Wkly Rep. 2020;69(32):1081-1088.

12. Ludvigsson JF. Systematic review of COVID-19 in children shows milder cases and a better prognosis than adults. Acta Paediatr. 2020;109(6):1088-1095.
13. Pierce $\mathrm{CA}$, et al. Immune responses to SARS-CoV-2 infection in hospitalized pediatric and adult patients. Sci Transl Med. 2020;12(564):eabd5487.

14. Riphagen S, Gomez X, Gonzalez-Martinez C, Wilkinson N, Theocharis P. Hyperinflammatory shock in children during COVID-19 pandemic. Lancet . 2020;395(10237):1607-1608.

15. Feldstein LR, et al. Multisystem inflammatory syndrome in U.S. children and adolescents. NEngl J Med. 2020;383(4):334-346.

16. Weisberg SP, et al. Antibody responses to SARS$\mathrm{CoV} 2$ are distinct in children with MIS-C compared to adults with COVID-19. medRxiv. Preprint posted online July 14, 2020. https://doi.org/ 10.1101/2020.07.12.20151068. Accessed October 28, 2020.

17. Consiglio CR, et al. The immunology of multisystem inflammatory syndrome in children with COVID-19. Cell. Preprint posted online September 6, 2020. https://doi.org/1016/j.cell.2020.09.016.

18. Whittaker E, et al. Clinical characteristics of 58 children with a pediatric inflammatory multisystem syndrome temporally associated with SARS-CoV-2. JAMA. 2020;324(3):259-269.

19. Godfred-Cato S, et al. COVID-19-associated multisystem inflammatory syndrome in children - United States, March-July 2020. MMWR Morb Mortal Wkly Rep. 2020;69(32):1074-1080.

20. Shaigany S, et al. An adult with Kawasaki-like multisystem inflammatory syndrome associated with COVID-19. Lancet. 2020;396(10246):e8-e10. 